\title{
A Generalized Henry-Type Integral Inequality and Application to Dependence on Orders and Known Functions for a Fractional Differential Equation
}

\author{
Jun Zhou ${ }^{1,2}$ \\ ${ }^{1}$ Department of Mathematics, Sichuan University, Chengdu, Sichuan 610064, China \\ ${ }^{2}$ College of Mathematics and Software Science, Sichuan Normal University, Chengdu, Sichuan 610066, China \\ Correspondence should be addressed to Jun Zhou; matzhj@126.com
}

Received 30 March 2014; Accepted 10 June 2014; Published 7 July 2014

Academic Editor: Turgut Öziş

Copyright ( 2014 Jun Zhou. This is an open access article distributed under the Creative Commons Attribution License, which permits unrestricted use, distribution, and reproduction in any medium, provided the original work is properly cited.

We discuss on integrable solutions for a generalized Henry-type integral inequality in which weak singularity and delays are involved. Not requiring continuity or differentiability for some given functions, we use a modified iteration argument to give an estimate of the unknown function in terms of the multiple Mittag-Leffler function. We apply the result to give continuous dependence of solutions on initial data, derivative orders, and known functions for a fractional differential equation.

\section{Introduction}

Since Gronwall [1] and Bellman [2] discussed the integral inequalities

$$
\begin{gathered}
0 \leq u(t) \leq \int_{\alpha}^{t}(a+b u(s)) d s, \\
u(t) \leq a+\int_{\alpha}^{t} f(s) u(s) d s,
\end{gathered}
$$

respectively, there have been made many generalizations, some of which were applied to existence, uniqueness, boundedness, and stability of solutions and invariant manifolds for differential equations and integral equations. In 1956 Bihari [3] discussed a nonlinear version of the integral inequality

$$
u(t) \leq a+\int_{0}^{t} f(s) w(u(s)) d s, \quad t \geq 0,
$$

where the given function $w$ is continuous, nondecreasing, and positive definite (i.e., $w(u) \geq 0$ for all $u$ and $w(u)=0$ if and only if $u=0)$ on $[0, \infty)$. In 2000 this result was generalized by Lipovan [4] to the delay case

$$
\begin{aligned}
u(t) \leq & a+\int_{t_{0}}^{t} f(s) w(u(s)) d s \\
& +\int_{b\left(t_{0}\right)}^{b(t)} g(s) w(u(s)) d s, \quad t_{0} \leq t<t_{1},
\end{aligned}
$$

where $b$ is a continuously differentiable and nondecreasing function from $\left[t_{0}, t_{1}\right)$ to $\left[t_{0}, t_{1}\right)$ such that $b(t) \leq t$. In 2005 Agarwal et al. [5] investigated the integral inequality of a finite sum

$$
\begin{array}{r}
u(t) \leq a(t)+\sum_{i=1}^{n} \int_{b_{i}\left(t_{0}\right)}^{b_{i}(t)} f_{i}(t, s) w_{i}(u(s)) d s, \\
t_{0} \leq t<t_{1},
\end{array}
$$

where the monotonicity of $a$ is not required but $w_{i+1}$ has stronger monotonicity than $w_{i}$ for each $i$. In recent years some new generalizations were given in, for example, [6-8]. Many results on integral inequalities can be found in Pachpatte's monograph (see [9]).

Among various types of integral inequalities, integral inequalities with weakly singular kernels are important tools 
in the discussion of reaction-diffusion equations and fractional differential equations. As shown in [10], the integral $\int_{t 0}^{t} K(t, s)$ is singular on the line $s=t$; it is referred to be weakly singular if it is singular and $\int_{t 0}^{t}|f(t, s)| d s<\infty$ for all $t_{0} \leq t<T \leq \infty$. In fractional differential equations we need to consider the following Riemann-Liouville derivative operator and integral operator (see [11])

$$
\begin{aligned}
D_{t_{0}}^{\alpha} f(x):= & \frac{1}{\Gamma(1-\alpha)} \frac{d}{d x} \\
& \times \int_{t_{0}}^{x}(x-t)^{-\alpha} f(t) d t, \quad 0<\alpha<1, \\
I_{t_{0}}^{\beta} f(x):= & \frac{1}{\Gamma(\beta)} \int_{t_{0}}^{x}(x-t)^{\beta-1} f(t) d t, \quad \beta>0,
\end{aligned}
$$

in which the singular kernels $(x-t)^{-\alpha}$ and $(x-t)^{\beta-1}$ are included, respectively. Another example can be seen from the Cauchy problem of the evolution equation

$$
\dot{x}+A(t) x=f(t), \quad x\left(t_{0}\right)=x_{0},
$$

in a Banach space $X$, where $A$ is a sectorial operator and $f$ is locally Hölder continuous with Hölder index $0<\theta<1$. From the variation-constant formula (see [12]), we encounter the following:

$$
\begin{gathered}
\left\|A e^{-A(t-s)}\right\|=O\left((t-s)^{-1}\right), \\
\|f(t)-f(s)\|=O\left((t-s)^{\theta}\right),
\end{gathered}
$$

and therefore the singular kernel $(t-s)^{\theta-1}$ is the estimation of its solution. For this reason the integral inequality with a weakly singular kernel

$$
u(t) \leq a(t)+b \int_{0}^{t}(t-s)^{\beta-1} u(s) d s, \quad t \geq 0
$$

where $b \geq 0,0<\beta<1$ and both $a$ and $u$ are nonnegative and locally integrable, was considered in Henry's book [12] in 1981 and the estimate

$$
u(t) \leq a(t)+\int_{0}^{t}\left\{\sum_{n=1}^{\infty} \frac{(b \Gamma(\beta))^{n}}{\Gamma(n \beta)}(t-s)^{n \beta-1} a(s)\right\} d s, \quad t \geq 0,
$$

where $\Gamma$ is the Gamma function, was given by an iteration approach. Following Henry's idea, in 1994 Sano and Kunimatsu [13] extended Henry's result to a more general integral inequality

$$
\begin{aligned}
u(t) \leq & c_{1}+c_{2} t^{\beta-1}+c_{3} \int_{0}^{t} u(s) d s \\
& +c_{4} \int_{0}^{t}(t-s)^{\beta-1} u(s) d s, \quad t \geq 0
\end{aligned}
$$

where $c_{1}, c_{2}, c_{3}, c_{4} \geq 0,0<\beta<1$. Inequality (9) was also extended by Ye et al. [14] in 2007 by replacing the constant $b$ with a nonnegative, nondecreasing, bounded, and continuous function $g(t)$.

Another idea for inequalities with a weakly singular kernel was introduced by Medved [15] in 1997 for the following Henry-Bihari type integral inequality:

$$
u(t) \leq a(t)+\int_{0}^{t}(t-s)^{\beta-1} F(s) w(u(s)) d s, \quad t \geq 0,
$$

where $0<\beta<1$. This inequality is of Bihari's form (3) with a weakly singular kernel. He applied the well-known Hölder inequality to separate the unknown $u$ from the singular kernel, that is,

$$
\begin{aligned}
u(t) \leq & a(t)+\int_{0}^{t}(t-s)^{\beta-1} e^{s} F(s) e^{-s} w(u(s)) d s \\
\leq & a(t)+\left(\int_{0}^{t}(t-s)^{p(\beta-1)} e^{p s} d s\right)^{1 / p} \\
& \times\left(\int_{0}^{t} F(s)^{q} e^{-q s} w(u(s))^{q} d s\right)^{1 / q}
\end{aligned}
$$

where $p, q>1$ are certain constants such that $1 / p+1 / q=1$, so that the inserted exponential factor $e^{s}$ makes the singular integral $\int_{0}^{t}(t-s)^{p(\beta-1)} e^{p s} d s$ convergent as $s \rightarrow t$ and the inequality is reduced to the classic Bihari's form (3). In 2002 Ma and Yang [16] improved Medved's method and gave an estimation to the Volterra-type integral inequality with a more general form of weakly singular kernel

$$
\begin{array}{r}
u(t) \leq a(t)+b(t) \int_{0}^{t}\left(t^{\sigma}-s^{\sigma}\right)^{\mu-1} s^{\tau-1} g(s) u(s) d s \\
+c(t) \int_{0}^{t}\left(t^{\alpha}-s^{\alpha}\right)^{\beta-1} s^{\gamma-1} f(s) w(u(s)) d s, \\
t \geq 0,
\end{array}
$$

where $\alpha, \beta, \gamma, \sigma, \mu, \tau \geq 0$. Recently Ma and Pečarić [17] also employed the separation approach to discuss another weakly singular integral inequality

$$
u^{p}(t) \leq a(t)+b(t) \int_{0}^{t}\left(t^{\alpha}-s^{\alpha}\right)^{\beta-1} s^{\gamma-1} f(s) u^{q}(s) d s,
$$

$$
t \geq 0
$$

under the condition $p \geq q \geq 0$.

In this paper we investigate the following integral inequality of finite sum:

$$
\begin{array}{r}
u(t) \leq a(t)+\sum_{i=1}^{m} \int_{b_{i}\left(t_{0}\right)}^{b_{i}(t)}\left(t-f_{i}(s)\right)^{\beta_{i}} h_{i}(t, s) u(s) d s, \\
t \geq 0
\end{array}
$$

in which weakly singular kernels and delays are involved. Not requiring continuity of $a$ or differentiability of $b_{i}$, we give an estimate for locally integrable $u$ in terms of the multiple Mittag-Leffler function (see [18]). We prefer Henry's iteration 
approach in our proof because the approach does not reduce the problem to the classic one so that no more continuity and differentiability are required. Our result generalizes the works made in [14-17] in some sense because of the more general form (16). Finally, we apply the result to give continuous dependence of solutions on initial data, derivative orders, and known functions for a fractional differential equation.

\section{Main Results}

For constants $t_{0}, T$ such that $t_{0}<T \leq+\infty$, consider inequality (16), where $a, b_{i}, f_{i}$ and $h_{i}$ are given nonnegative functions and satisfy the following hypotheses:

$\left(\mathrm{H}_{1}\right) a$ is a locally integrable function on $\left[t_{0}, T\right)$, that is, Lebesgue integrable on every compact subset of $\left[t_{0}, T\right)$, and the integrations in (16) are bounded by replacing $u$ with $a$ in (16);

$\left(\mathrm{H}_{2}\right)$ every $h_{i}, i=1, \ldots, m$, is continuous on $\left[t_{0}, T\right) \times\left[t_{0}, T\right)$;

$\left(\mathrm{H}_{3}\right)$ every $b_{i}:\left[t_{0}, T\right) \rightarrow\left[t_{0}, T\right), i=1, \ldots, m$, is continuous and strictly increasing such that $b_{i}(t) \leq t$ for all $t \in\left[t_{0}, T\right)$;

$\left(\mathrm{H}_{4}\right)$ every $f_{i}, i=1, \ldots, m$, is continuously differentiable on $\left[t_{0}, T\right)$ such that $f_{i}^{\prime}(t)>0$ and $t \leq f_{i}(t) \leq b_{i}^{-1}(t)$ for all $t \in\left[t_{0}, T\right)$.

Theorem 1. Suppose that $\left(H_{1}\right)-\left(H_{4}\right)$ hold and $\beta_{i}>-1$, $i=1, \ldots, m$. Then, every nonnegative and locally integrable function $u(t)$ which satisfies (16) on $\left[t_{0}, T\right)$ and the integrations in (16) bounded has the estimate

$$
\begin{array}{r}
u(t) \leq a(t) \\
+\sum_{n=1}^{\infty}\left\{\sum_{1 \leq k_{1}, \ldots, k_{n} \leq m} \frac{\prod_{i=1}^{n} \Gamma\left(\beta_{k_{i}}+1\right) \tilde{h}_{k_{i}}(t)}{\Gamma\left(\sum_{i=1}^{n} \beta_{k_{i}}+n\right)}\right. \\
\cdot \int_{b_{k_{1}}\left(t_{0}\right)}^{b_{k_{1}}(t)}\left(t-f_{k_{1}}(s)\right)^{\sum_{i=1}^{n} \beta_{k_{i}}+n-1} \\
\left.\times f_{k_{1}}^{\prime}(s) a(s) d s\right\}, \quad t \in\left[t_{0}, T\right),
\end{array}
$$

where $\widetilde{h}_{i}(t)=\max _{(s, \tau) \in\left[t_{0}, t\right] \times\left[t_{0}, t\right]} h_{i}(s, \tau) / f_{i}^{\prime}(\tau), i=1, \ldots, m$.

We leave the proof of this theorem to next section. In what follows, we express the estimate of series form in terms of the multiple Mittag-Leffler function (see [18]).

The original Mittag-Leffler function

$$
E_{\alpha}(z):=\sum_{k=0}^{\infty} \frac{z^{k}}{\Gamma(k \alpha+1)}, \quad z \in \mathbb{C}, \alpha>0
$$

was proposed as an extension of the exponential function by Mittag-Leffler ([19]) in 1903. An extension of two parameters

$$
E_{\alpha, \beta}(z):=\sum_{k=0}^{\infty} \frac{z^{k}}{\Gamma(k \alpha+\beta)}, \quad \alpha, \beta, z \in \mathbb{C}, \mathfrak{R}(\alpha), \mathfrak{R}(\beta)>0,
$$

was proposed by Wiman [20] in 1905. Later, two MittagLeffler functions with three parameters were given separately by Prabhakar [21] and Kilbas and Saigo [22]. In 1996 Hadid and Luchko [18] generalized the function into the multiple form

$$
E_{\left(\alpha_{1}, \ldots, \alpha_{n}\right), \beta}\left(z_{1}, \ldots, z_{n}\right):=\sum_{k=0}^{\infty} \sum_{\substack{l_{1}+\cdots+l_{n}=k \\ l_{1}, \ldots, l_{n} \geq 0}} \frac{\left(k ; l_{1}, \ldots, l_{n}\right) \prod_{i=1}^{n} z_{i}^{l_{i}}}{\Gamma\left(\sum_{i=1}^{n} l_{i} \alpha_{i}+\beta\right)},
$$

where $\alpha_{i}, \beta, z_{i} \in \mathbb{C}, \mathfrak{R}\left(\alpha_{i}\right), \mathfrak{R}(\beta)>0, i=1, \ldots, n$ and

$$
\left(k ; l_{1}, \ldots, l_{n}\right):=\frac{k !}{l_{1} ! \times \cdots \times l_{n} !} .
$$

These generalized Mittag-Leffler functions have been treated as significant special functions since they played an important role in computing fractional calculus and solving fractional differential and integral equations modeled in physics, chemistry, biology, engineering, and applied sciences (see monographs $[11,23])$. We have the following inequality:

$$
\begin{array}{r}
E_{\left(\alpha_{1}, \ldots, \alpha_{n}\right), 1}\left(z_{1}, \ldots, z_{n}\right) \leq \prod_{i=1}^{n} E_{\alpha_{i}}\left(z_{i}\right), \\
\alpha_{i}, z_{i} \in \mathbb{R}_{+}, \quad i=1, \ldots, n .
\end{array}
$$

In fact, we can show that

$$
\Gamma\left(\sum_{i=1}^{m} a_{i}+1\right) \geq \prod_{i=1}^{m} \Gamma\left(a_{i}+1\right), \quad a_{i}>0, i=1, \ldots, m .
$$

By Euler's definition on Gamma function (see [24]), we have

$$
\begin{gathered}
\Gamma\left(a_{i}+1\right)=\frac{1}{a_{i}+1} \prod_{n=1}^{\infty} \frac{(1+(1 / n))^{a_{i}+1}}{1+\left(\left(a_{i}+1\right) / n\right)}, \\
\Gamma\left(\sum_{i=1}^{m} a_{i}+1\right)=\frac{1}{\sum_{i=1}^{m} a_{i}+1} \prod_{n=1}^{\infty} \frac{(1+(1 / n))^{\sum_{i=1}^{m} a_{i}+1}}{1+\left(\sum_{i=1}^{m}\left(a_{i}+1\right) / n\right)} .
\end{gathered}
$$

It follows that

$$
\frac{\Gamma\left(\sum_{i=1}^{m} a_{i}+1\right)}{\prod_{i=1}^{m} \Gamma\left(a_{i}+1\right)}
$$

$$
=\frac{\prod_{i=1}^{m}\left(a_{i}+1\right)}{\sum_{i=1}^{m} a_{i}+1} \prod_{n=1}^{\infty} \frac{\prod_{i=1}^{m}\left(1+\left(\left(a_{i}+1\right) / n\right)\right)}{(1+(1 / n))^{m-1}\left(1+\left(\sum_{i=1}^{m}\left(a_{i}+1\right) / n\right)\right)} .
$$

It is easy to know that $\prod_{i=1}^{m}\left(a_{i}+1\right) \geq \sum_{i=1}^{m} a_{i}+1$. Then, we can prove by induction that

$$
\prod_{i=1}^{m}\left(1+\frac{a_{i}+1}{n}\right) \geq\left(1+\frac{1}{n}\right)^{m-1}\left(1+\frac{\sum_{i=1}^{m} a_{i}+1}{n}\right) .
$$


Obviously, (26) is true for $m=1$. Assume that (26) is true for $m=l$. We get

$$
\begin{aligned}
\prod_{i=1}^{l+1}\left(1+\frac{a_{i}+1}{n}\right)= & \left(1+\frac{1}{n}\right)^{l-1}\left(1+\frac{\sum_{i=1}^{l} a_{i}+1}{n}\right) \\
& \times\left(1+\frac{a_{l+1}+1}{n}\right) \\
\geq & \left(1+\frac{1}{n}\right)^{l}\left(1+\frac{\sum_{i=1}^{l+1} a_{i}+1}{n}\right),
\end{aligned}
$$

since

$$
\begin{array}{r}
\left(1+\frac{\sum_{i=1}^{l} a_{i}+1}{n}\right)\left(1+\frac{a_{l+1}+1}{n}\right) \\
\geq\left(1+\frac{1}{n}\right)\left(1+\frac{\sum_{i=1}^{l+1} a_{i}+1}{n}\right),
\end{array}
$$

implying that (26) holds for $m=l+1$. Thus, from (26) we see that (23) holds. By (23), we have

$$
\begin{aligned}
& E_{\left(\alpha_{1}, \ldots, \alpha_{n}\right), 1}\left(z_{1}, \ldots, z_{n}\right) \\
& \quad=\sum_{m=0}^{\infty} \sum_{1 \leq k_{1}, \ldots, k_{m} \leq n} \frac{\prod_{i=1}^{m} z_{k_{i}}}{\Gamma\left(\sum_{i=1}^{m} \alpha_{k_{i}}+1\right)} \\
& \quad \leq \sum_{k_{1}=0}^{\infty} \ldots \sum_{k_{n}=0}^{\infty} \frac{\prod_{i=1}^{n} z_{i}^{k_{i}}}{\prod_{i=1}^{n} \Gamma\left(k_{i} \alpha_{i}+1\right)}=\prod_{i=1}^{n} E_{\alpha_{i}}\left(z_{i}\right) .
\end{aligned}
$$

Therefore, (22) is proved.

Corollary 2. Suppose that the hypotheses of Theorem 1 hold and additionally that $a(t)$ is continuous on $\left[t_{0}, T\right)$. Then,

$$
\begin{aligned}
u(t) \leq & \tilde{a}(t) E_{\left(\beta_{1}+1, \ldots, \beta_{m}+1\right), 1} \\
& \times\left(\widetilde{h}_{1}(t) \Gamma\left(\beta_{1}+1\right)\left(t-t_{0}\right)^{\beta_{1}+1},\right. \\
& \left.\ldots, \widetilde{h}_{m}(t) \Gamma\left(\beta_{m}+1\right)\left(t-t_{0}\right)^{\beta_{m}+1}\right) \\
\leq & \widetilde{a}(t) \prod_{i=1}^{m} E_{\beta_{i}+1}\left(\widetilde{h}_{i}(t) \Gamma\left(\beta_{i}+1\right)\left(t-t_{0}\right)^{\beta_{i}+1}\right),
\end{aligned}
$$

where $\tilde{a}(t)=\max _{t_{0} \leq \tau \leq t} a(\tau)$ and $E_{\alpha}(z)$ and $E_{\left(\alpha_{1}, \ldots, \alpha_{n}\right), 1}$ are defined in (18) and (20).

Proof. Starting from (17), we have

$$
\begin{array}{r}
u(t) \leq \widetilde{a}(t)\left\{1+\sum_{n=1}^{\infty}\left\{\sum_{1 \leq k_{1}, \ldots, k_{n} \leq m} \frac{\prod_{i=1}^{n} \Gamma\left(\beta_{k_{i}}+1\right) \tilde{h}_{k_{i}}(t)}{\Gamma\left(\sum_{i=1}^{n} \beta_{k_{i}}+n\right)}\right.\right. \\
\times \int_{b_{k_{1}}\left(t_{0}\right)}^{b_{k_{1}}(t)}\left(t-f_{k_{1}}(s)\right)^{\sum_{i=1}^{n} \beta_{k_{i}}+n-1} \\
\left.\left.\times f_{k_{1}}^{\prime}(s) d s\right\}\right\}
\end{array}
$$

$$
\begin{aligned}
& \leq \widetilde{a}(t)\left\{1+\sum_{n=1}^{\infty}\left(\sum_{1 \leq k_{1}, \ldots, k_{n} \leq m} \frac{\prod_{i=1}^{n} \Gamma\left(\beta_{k_{i}}+1\right) \tilde{h}_{k_{i}}(t)}{\Gamma\left(\sum_{i=1}^{n} \beta_{k_{i}}+n+1\right)}\right.\right. \\
& \left.\left.\times\left(t-t_{0}\right)^{\sum_{i=1}^{n}\left(\beta_{k_{i}}+1\right)}\right)\right\} \\
& =\tilde{a}(t) \sum_{n=0}^{\infty} \sum_{1 \leq k_{1}, \ldots, k_{n} \leq m}\left(\prod_{i=1}^{n} \Gamma\left(\beta_{k_{i}}+1\right) \tilde{h}_{k_{i}}(t)\right. \\
& \left.\times\left(t-t_{0}\right)^{\beta_{k_{i}}+1}\right) \\
& \times\left(\Gamma\left(\sum_{i=1}^{n} \beta_{k_{i}}+n+1\right)\right)^{-1} \\
& =\widetilde{a}(t) \\
& \times \sum_{k=0}^{\infty} \sum_{\substack{k_{1}+\cdots+k_{m}=k \\
k_{1}, \ldots, k_{m} \geq 0}}\left(\left(k ; k_{1}, \ldots, k_{m}\right)\right. \\
& \times \prod_{i=1}^{m}\left(\Gamma\left(\beta_{i}+1\right) \tilde{h}_{i}(t)\right. \\
& \left.\left.\times\left(t-t_{0}\right)^{\beta_{i}+1}\right)^{k_{i}}\right) \\
& \times\left(\Gamma\left(\sum_{i=1}^{m} k_{i}\left(\beta_{i}+1\right)+1\right)\right)^{-1} \\
& =\tilde{a}(t) E_{\left(\beta_{1}+1, \ldots, \beta_{m}+1\right), 1} \\
& \times\left(\tilde{h}_{1}(t) \Gamma\left(\beta_{1}+1\right)\left(t-t_{0}\right)^{\beta_{1}+1},\right. \\
& \left.\ldots, \tilde{h}_{m}(t) \Gamma\left(\beta_{m}+1\right)\left(t-t_{0}\right)^{\beta_{m}+1}\right) .
\end{aligned}
$$

It follows from (22) that

$$
\begin{gathered}
\widetilde{a}(t) E_{\left(\beta_{1}+1, \ldots, \beta_{m}+1\right), 1} \\
\times\left(\widetilde{h}_{1}(t) \Gamma\left(\beta_{1}+1\right)\left(t-t_{0}\right)^{\beta_{1}+1},\right. \\
\left.\ldots, \tilde{h}_{m}(t) \Gamma\left(\beta_{m}+1\right)\left(t-t_{0}\right)^{\beta_{m}+1}\right) \\
\leq \tilde{a}(t) \prod_{i=1}^{m} E_{\beta_{i}+1}\left(\tilde{h}_{i}(t) \Gamma\left(\beta_{i}+1\right)\left(t-t_{0}\right)^{\beta_{i}+1}\right) .
\end{gathered}
$$

The corollary is proved.

\section{Proof of Theorem}

Let $L_{\text {loc }, w}^{1}\left[t_{0}, T\right)$ consist of all locally integrable nonnegative functions $\phi$ on $\left[t_{0}, T\right)$ such that $\sum_{i=1}^{m} \int_{b_{i}\left(t_{0}\right)}^{b_{i}(t)}\left(t-f_{i}(s)\right)^{\beta_{i}}$ $h_{i}(t, s) \phi(s) d s<\infty$ for arbitrarily given $t \in\left[t_{0}, T\right)$. We can 
verify that $L_{\text {loc, } w}^{1}\left[t_{0}, T\right)$ is a linear space, due to the linearity of integration. Define a linear operator $B$ on $L_{\text {loc }, w}^{1}\left[t_{0}, T\right)$ as

$$
B \phi(t):=\sum_{i=1}^{m} \int_{b_{i}\left(t_{0}\right)}^{b_{i}(t)}\left(t-f_{i}(s)\right)^{\beta_{i}} h_{i}(t, s) \phi(s) d s, \quad t \in\left[t_{0}, T\right),
$$

where $\phi \in L_{\mathrm{loc}, w}^{1}\left[t_{0}, T\right)$. We claim that $B$ is self-mapping on $L_{\text {loc }, w}^{1}\left[t_{0}, T\right)$. In fact, for all $\phi \in L_{\text {loc }, w}^{1}\left[t_{0}, T\right)$ and given $t \in$ $\left[t_{0}, T\right), \sum_{i=1}^{m} \int_{b_{i}\left(t_{0}\right)}^{b_{i}(t)}\left(t-f_{i}(s)\right)^{\beta_{i}} h_{i}(t, s) \phi(s) d s<\infty$, implying that $B \phi \in C_{+}\left[t_{0}, T\right)$, the set of all continuous and nonnegative functions on $\left[t_{0}, T\right)$. We know that $C_{+}\left[t_{0}, T\right) \quad \mathrm{c}$ $L_{\text {loc }}^{1}\left[t_{0}, T\right)$. For all $\psi \in C_{+}\left[t_{0}, T\right)$, from the continuity of $\psi$ and $h_{i}$, we know that they are all locally bounded. We also know that $\left(t-f_{i}(s)\right)^{\beta_{i}}$ are integrable on $s \in$ $\left[b_{i}\left(t_{0}\right), b_{i}(t)\right]$ by $\left(\mathrm{H}_{3}\right),\left(\mathrm{H}_{4}\right)$ and $\beta_{i}>-1$. It follows that $\sum_{i=1}^{m} \int_{b_{i}\left(t_{0}\right)}^{b_{i}(t)}\left(t-f_{i}(s)\right)^{\beta_{i}} h_{i}(t, s) \psi(s) d s<\infty$, implying that $C_{+}\left[t_{0}, T\right) \subset L_{\text {loc } w}^{1}\left[t_{0}, T\right)$. Then, inequality (16) can be simplified as $u(t) \leq a(t)+B u(t)$, from which we can prove by induction that

$$
u(t) \leq \sum_{l=0}^{n-1} B^{l} a(t)+B^{n} u(t)
$$

We claim that for all integers $n \geq 1$,

$$
\begin{aligned}
B^{n} u(t) \leq & \sum_{1 \leq k_{1}, \ldots, k_{n} \leq m} \frac{\prod_{i=1}^{n} \Gamma\left(\beta_{k_{i}}+1\right) \tilde{h}_{k_{i}}(t)}{\Gamma\left(\sum_{i=1}^{n} \beta_{k_{i}}+n\right)} \\
& \cdot \int_{b_{k_{n}}\left(t_{0}\right)}^{b_{k_{n}}(t)}\left(t-f_{k_{n}}(s)\right)^{\sum_{i=1}^{n} \beta_{k_{i}}+n-1} f_{k_{n}}^{\prime}(s) u(s) d s,
\end{aligned}
$$

where $\widetilde{h}_{k_{i}}$ are well-defined on $\left[t_{0}, T\right)$ since $h_{k_{i}}$ and $f_{k_{i}}^{\prime}$ are continuous and $f_{k_{i}}^{\prime}(t)>0$ on $\left[t_{0}, T\right)$. In fact, (35) is true for $n=1$. Assume that (35) is true for $n=l$. Then

$$
\begin{array}{r}
B^{l+1} u(t)=B^{l}(B u(t)) \\
\leq \sum_{1 \leq k_{1}, \ldots, k_{l} \leq m} \frac{\prod_{i=1}^{l} \Gamma\left(\beta_{k_{i}}+1\right) \tilde{h}_{k_{i}}(t)}{\Gamma\left(\sum_{i=1}^{l} \beta_{k_{i}}+l\right)} \\
\quad \times \int_{b_{k_{l}}\left(t_{0}\right)}^{b_{k_{k}}(t)}\left(t-f_{k_{l}}(s)\right)^{\sum_{i=1}^{l} \beta_{k_{i}}+l-1} f_{k_{l}}^{\prime}(s) \\
\cdot \sum_{k_{l+1}=1}^{m}\left\{\int_{b_{k_{l+1}}\left(t_{0}\right)}^{b_{k_{l+1}}(s)} h_{k_{l+1}}(s, \tau)\right. \\
\times\left(s-f_{k_{l+1}}(\tau)\right)^{\beta_{k_{l+1}}} \\
\times u(\tau) d \tau\} d s .
\end{array}
$$

It follows that

$$
\begin{aligned}
B^{l+1} u(t) \leq & \sum_{1 \leq k_{1}, \ldots, k_{l} \leq m} \frac{\prod_{i=1}^{l} \Gamma\left(\beta_{k_{i}}+1\right) \widetilde{h}_{k_{i}}(t)}{\Gamma\left(\sum_{i=1}^{l} \beta_{k_{i}}+l\right)} \\
& \times \sum_{k_{l+1}=1}^{m} \widetilde{h}_{k_{l+1}}(t) \Phi(t),
\end{aligned}
$$

where

$$
\begin{gathered}
\Phi(t):=\int_{b_{k_{l}}\left(t_{0}\right)}^{b_{k_{l}}(t)}\left(t-f_{k_{l}}(s)\right)^{\sum_{i=1}^{l} \beta_{k_{i}}+l-1} f_{k_{l}}^{\prime}(s) \varphi(s) d s, \\
\varphi(s):=\int_{b_{k_{l+1}}\left(t_{0}\right)}^{b_{k_{l+1}}(s)}\left(s-f_{k_{l+1}}(\tau)\right)^{\beta_{k_{l+1}}} f_{k_{l+1}}^{\prime}(\tau) u(\tau) d \tau .
\end{gathered}
$$

Note that $f_{k_{i}}\left(b_{k_{i}}(t)\right) \leq t$ by $\left(\mathrm{H}_{4}\right)$. It implies that $s-f_{k_{l+1}}(\tau) \geq 0$ for all $\tau \in\left[b_{k_{l+1}}\left(t_{0}\right), b_{k_{l+1}}(s)\right]$ by the monotonicity of $f_{k_{l+1}}$. It follows from (39) that $\varphi$ is a nondecreasing function. Hence, $\varphi\left(f_{k_{l}}^{-1}(t)\right) \leq \varphi(t)$ by $\left(\mathrm{H}_{4}\right)$. On the other hand, from $\left(\mathrm{H}_{3}\right)$ and $\left(\mathrm{H}_{4}\right)$ we see that $b_{i}\left(t_{0}\right)=f_{i}\left(t_{0}\right)=t_{0}$, implying that $f_{k_{l}}\left(b_{k_{l}}\left(t_{0}\right)\right)=t_{0}$. With the change of variables $\xi=f_{k_{l}}(s)$, it follows from (38) that

$$
\begin{aligned}
\Phi(t) & =\int_{f_{k_{l}}\left(b_{k_{l}}\left(t_{0}\right)\right)}^{f_{k_{l}}\left(b_{k_{l}}(t)\right)}(t-\xi)^{\sum_{i=1}^{l} \beta_{k_{i}}+l-1} \varphi\left(f_{k_{l}}^{-1}(\xi)\right) d \xi \\
& \leq \int_{t_{0}}^{t}(t-\xi)^{\sum_{i=1}^{l} \beta_{k_{i}}+l-1} \varphi\left(f_{k_{l}}^{-1}(\xi)\right) d \xi \\
& \leq \int_{t_{0}}^{t}(t-\xi)^{\sum_{i=1}^{l} \beta_{k_{i}}+l-1} \varphi(\xi) d \xi .
\end{aligned}
$$

Letting $s$ denote $\xi$ and substituting (39) in (40), we get

$$
\begin{gathered}
\Phi(t) \leq \int_{t_{0}}^{t} \int_{b_{k_{l+1}}\left(t_{0}\right)}^{b_{k_{l+1}}(s)}(t-s)^{\sum_{i=1}^{l} \beta_{k_{i}}+l-1}\left(s-f_{k_{l+1}}(\tau)\right)^{\beta_{k_{l+1}}} \\
\times f_{k_{l+1}}^{\prime}(\tau) u(\tau) d \tau d s \\
=\int_{b_{k_{l+1}}\left(t_{0}\right)}^{b_{k_{l+1}}(t)} \int_{b_{k_{l+1}}^{-1}(\tau)}^{t}(t-s)^{\sum_{i=1}^{l} \beta_{k_{i}}+l-1} \\
\times\left(s-f_{k_{l+1}}(\tau)\right)^{\beta_{k_{l+1}}} d s \\
\times f_{k_{l+1}}^{\prime}(\tau) u(\tau) d \tau
\end{gathered}
$$

by interchanging the order of integration. In (41) we observe that

$$
\begin{aligned}
& \int_{b_{k_{l+1}}^{-1}(\tau)}^{t}(t-s)^{\sum_{i=1}^{l} \beta_{k_{i}}+l-1}\left(s-f_{k_{l+1}}(\tau)\right)^{\beta_{k_{l+1}}} d s \\
& \quad=\left(t-f_{k_{l+1}}(\tau)\right)^{\sum_{i=1}^{l+1} \beta_{k_{i}}+l}
\end{aligned}
$$




$$
\begin{aligned}
& \quad \times \int_{b_{k_{l+1}}^{-1}(\tau)}^{t}\left(\frac{t-s}{t-f_{k_{l+1}}(\tau)}\right)^{\sum_{i=1}^{l} \beta_{k_{i}}+l-1} \\
& \quad \times\left(\frac{s-f_{k_{l+1}}(\tau)}{t-f_{k_{l+1}}(\tau)}\right)^{\beta_{k_{l+1}}} d\left(\frac{s-f_{k_{l+1}}(\tau)}{t-f_{k_{l+1}}(\tau)}\right) \\
& =\left(t-f_{k_{l+1}}(\tau)\right)^{\sum_{i=1}^{l+1} \beta_{k_{i}}+l} \\
& \quad \times \int_{z_{1}}^{1}(1-z)^{\sum_{i=1}^{l} \beta_{k_{i}}+l-1} z^{\beta_{k_{l+1}}} d z \\
& \leq\left(t-f_{k_{l+1}}(\tau)\right)^{\sum_{i=1}^{l+1} \beta_{k_{i}}+l} \\
& \quad \times \int_{0}^{1}(1-z)^{\sum_{i=1}^{l} \beta_{k_{i}}+l-1} z^{\beta_{k_{l+1}}} d z \\
& =\left(t-f_{k_{l+1}}(\tau)\right)^{\sum_{i=1}^{l+1} \beta_{k_{i}}+l} B\left(\sum_{i=1}^{l} \beta_{k_{i}}+l, \beta_{k_{l+1}}+1\right) \\
& =\frac{\Gamma\left(\sum_{i=1}^{l} \beta_{k_{i}}+l\right) \Gamma\left(\beta_{k_{l+1}}+1\right)}{\Gamma\left(\sum_{i=1}^{l+1} \beta_{k_{i}}+l+1\right)} \\
& \quad \times\left(t-f_{k_{l+1}}(\tau)\right)^{\sum_{i=1}^{l+1} \beta_{k_{i}}+l},
\end{aligned}
$$

where $z:=\left\{s-f_{k_{l+1}}(\tau)\right\} /\left\{t-f_{k_{l+1}}(\tau)\right\}$ and $z_{1}:=\left\{b_{k_{l+1}}^{-1}(\tau)-\right.$ $\left.f_{k_{l+1}}(\tau)\right\} /\left\{t-f_{k_{l+1}}(\tau)\right\} \geq 0$ by $\left(\mathrm{H}_{4}\right)$. Thus, from (37) we see that inequality (35) holds for $n=l+1$ and the claimed (35) is proved.

For every $\phi(t) \in L_{\mathrm{loc}, \omega}^{1}\left[t_{0}, T\right)$ and each $n=0,1, \ldots, \infty$, define a sequence of linear operators $A_{n}: L_{\text {loc }, w}^{1}\left[t_{0}, T\right) \rightarrow$ $C\left[t_{0}, T\right)$ by

$$
\begin{aligned}
& A_{n} \phi(t):= \sum_{1 \leq k_{1}, \ldots, k_{n} \leq m} \frac{\prod_{i=1}^{n} \Gamma\left(\beta_{k_{i}}+1\right) \widetilde{h}_{k_{i}}(t)}{\Gamma\left(\sum_{i=1}^{n} \beta_{k_{i}}+n\right)} \\
& \cdot \int_{b_{k_{n}}\left(t_{0}\right)}^{b_{k_{k}}(t)}\left(t-f_{k_{n}}(s)\right)^{\sum_{i=1}^{n} \beta_{k_{i}}+n-1} \\
& \times f_{k_{n}}^{\prime}(s) \phi(s) d s,
\end{aligned}
$$

where $\int_{b_{k_{n}}\left(t_{0}\right)}^{b_{k_{n}}(t)}\left(t-f_{k_{n}}(s)\right)^{\sum_{i=1}^{n} \beta_{k_{i}}+n-1} f_{k_{n}}^{\prime}(s) \phi(s) d s<\infty$ since $\int_{b_{k_{n}}\left(t_{0}\right)}^{b_{k^{\prime}}(t)}\left(t-f_{k_{n}}(s)\right)^{\beta_{k_{n}}} \phi(s) d s<\infty$ from the supposition of Theorem 1 and $\left(t-f_{k_{n}}(s)\right)^{\sum_{i=1}^{n-1}\left(\beta_{k_{i}}+1\right)} f_{k_{n}}^{\prime}(s)$ are continuous on $\left[b_{k_{n}}\left(t_{0}\right), b_{k_{n}}(t)\right)$. It implies from (35) that

$$
B^{n} \phi(t) \leq A_{n} \phi(t) .
$$

In what follows we will prove that for all $\phi \in L_{\text {loc }, w}^{1}\left[t_{0}, T\right)$ and arbitrarily given $t \in\left[t_{0}, T\right), \sum_{n=1}^{\infty} A_{n} \phi(t)$ converges. Define

$$
\Psi_{a}(x):=\frac{\Gamma(x)}{\Gamma(x+a)},
$$

where $x \in(0, \infty)$ and $a>0$ is a constant. By Euler's definition on Gamma function (see [24]), $\Gamma(x)=(1 / x) \prod_{n=1}^{\infty}$ $(1+(1 / n))^{x} /(1+(x / n))$, we know that

$$
\Psi_{a}(x)=\left(1+\frac{a}{x}\right) \prod_{n=1}^{\infty} \frac{1}{(1+(1 / n))^{a}}\left(1+\frac{a}{n+x}\right),
$$

and therefore $\Psi_{a}$ is strictly decreasing on $(0, \infty)$. Thus, for all integers $1 \leq k_{i}, k_{j} \leq m, 1 \leq i, j \leq n$ and $\delta \geq 0$ satisfying that $-1<\beta_{k_{j}}-\delta<0$, we have $\Psi_{a}\left(\beta_{k_{j}}+1\right) \leq \Psi_{a}\left(\beta_{k_{j}}-\delta+1\right)$, where $a:=\left(\sum_{i=1}^{n} \beta_{k_{i}}\right)+n-\beta_{k_{j}}-1$, implying that

$$
\frac{\Gamma\left(\beta_{k_{j}}+1\right)}{\Gamma\left(\sum_{i=1}^{n} \beta_{k_{i}}+n\right)} \leq \frac{\Gamma\left(\beta_{k_{j}}-\delta+1\right)}{\Gamma\left(\sum_{i=1}^{n} \beta_{k_{i}}-\delta+n\right)} .
$$

Multiplying the above inequality by $\left(\prod_{i=1}^{n} \Gamma\left(\beta_{k_{i}}+1\right)\right) / \Gamma\left(\beta_{k_{j}}+\right.$ 1), we get that

$$
\begin{aligned}
& \frac{\prod_{i=1}^{n} \Gamma\left(\beta_{k_{i}}+1\right)}{\Gamma\left(\sum_{i=1}^{n} \beta_{k_{i}}+n\right)} \\
& \quad \leq \frac{\prod_{i=1}^{j-1} \Gamma\left(\beta_{k_{i}}+1\right) \Gamma\left(\beta_{k_{j}}-\delta+1\right) \prod_{i=j+1}^{n} \Gamma\left(\beta_{k_{i}}+1\right)}{\Gamma\left(\sum_{i=1}^{n} \beta_{k_{i}}-\delta+n\right)} .
\end{aligned}
$$

Let $\beta_{*}:=\min _{1 \leq i \leq m} \beta_{i}, \beta^{*}:=\max _{1 \leq i \leq m} \beta_{i}$ and $c:=\left(\beta^{*}+\right.$ $1) /\left(\beta_{*}+1\right) \geq 1$. Then (48) implies that

$$
\frac{\prod_{i=1}^{n} \Gamma\left(\beta_{k_{i}}+1\right)}{\Gamma\left(\sum_{i=1}^{n} \beta_{k_{i}}+n\right)} \leq \frac{\left(\Gamma\left(\beta_{*}+1\right)\right)^{n}}{\Gamma\left(n\left(\beta_{*}+1\right)\right)} .
$$

When $t \in\left(t_{0}+1, T\right)$, we have

$$
\begin{aligned}
& A_{n} \phi(t)= \sum_{1 \leq k_{1}, \ldots, k_{n} \leq m} \frac{\prod_{i=1}^{n} \Gamma\left(\beta_{k_{i}}+1\right) \tilde{h}_{k_{i}}(t)}{\Gamma\left(\sum_{i=1}^{n} \beta_{k_{i}}+n\right)} \\
& \times \int_{b_{k_{n}}\left(t_{0}\right)}^{b_{k_{n}}(t)}\left(t-f_{k_{n}}(s)\right)^{\sum_{i=1}^{n} \beta_{k_{i}}+n-1} f_{k_{n}}^{\prime}(s) \phi(s) d s \\
&= \sum_{1 \leq k_{1}, \ldots, k_{n} \leq m} \frac{\prod_{i=1}^{n} \Gamma\left(\beta_{k_{i}}+1\right) \widetilde{h}_{k_{i}}(t)}{\Gamma\left(\sum_{i=1}^{n} \beta_{k_{i}}+n\right)} \\
& \times\left\{\int_{b_{k_{n}}\left(t_{0}\right)}^{f_{k_{n}}^{-1}(t-1)}\left(t-f_{k_{n}}(s)\right)^{\beta_{k_{n}}} \phi(s)\right. \\
& \quad \times\left(t-f_{k_{n}}(s)\right)^{\sum_{i=1}^{n-1} \beta_{k_{i}}+n-1} f_{k_{n}}^{\prime}(s) d s \\
&+\int_{f_{k_{n}}^{-1}(t-1)}^{b_{k_{n}}(t)}\left(t-f_{k_{n}}(s)\right)^{\beta_{k_{n}}} \phi(s) \\
&\left.\times\left(t-f_{k_{n}}(s)\right)^{\sum_{i=1}^{n-1} \beta_{k_{i}}+n-1} f_{k_{n}}^{\prime}(s) d s\right\}
\end{aligned}
$$




$$
\begin{aligned}
& \leq m^{n-1}\left(\max _{i=1, \ldots, m}\left\{\tilde{h}_{i}(t)\right\}\right)^{n} \frac{\left(\Gamma\left(\beta_{*}+1\right)\right)^{n}}{\Gamma\left(n\left(\beta_{*}+1\right)\right)} \\
& \times \sum_{i=1}^{m}\left\{\int_{b_{i}\left(t_{0}\right)}^{f_{i}^{-1}(t-1)}\left(t-f_{i}(s)\right)^{\beta_{i}} \phi(s)\right. \\
& \times\left(t-f_{i}(s)\right)^{(n-1) c\left(\beta_{*}+1\right)} f_{i}^{\prime}(s) d s \\
& +\int_{f_{i}^{-1}(t-1)}^{b_{i}(t)}\left(t-f_{i}(s)\right)^{\beta_{i}} \phi(s) \\
& \left.\times\left(t-f_{i}(s)\right)^{(n-1)\left(\beta_{*}+1\right)} f_{i}^{\prime}(s) d s\right\} \\
& \leq\left(m \max _{i=1, \ldots, m}\left\{\tilde{h}_{i}(t)\right\}\right)^{n} \frac{\left(\Gamma\left(\beta_{*}+1\right)\right)^{n}}{\Gamma\left(n\left(\beta_{*}+1\right)\right)} \\
& \times\left\{\left(t-t_{0}\right)^{(n-1) c\left(\beta_{*}+1\right)}\right. \\
& \times \int_{b_{j}\left(t_{0}\right)}^{f_{j}^{-1}(t-1)}\left(t-f_{j}(s)\right)^{\beta_{j}} \phi(s) f_{j}^{\prime}(s) d s \\
& \left.+\int_{f_{j}^{-1}(t-1)}^{b_{j}(t)}\left(t-f_{j}(s)\right)^{\beta_{j}} \phi(s) f_{j}^{\prime}(s) d s\right\} \\
& \leq\left(m \max _{i=1, \ldots, m}\left\{\widetilde{h}_{i}(t)\right\}\right)^{n} \frac{\left(\Gamma\left(\beta_{*}+1\right)\right)^{n}}{\Gamma\left(n\left(\beta_{*}+1\right)\right)} \\
& \times\left(t-t_{0}\right)^{(n-1) c\left(\beta_{*}+1\right)} \\
& \times \int_{b_{j}\left(t_{0}\right)}^{b_{j}(t)}\left(t-f_{j}(s)\right)^{\beta_{j}} \phi(s) f_{j}^{\prime}(s) d s,
\end{aligned}
$$

where

$$
\begin{aligned}
& \int_{b_{j}\left(t_{0}\right)}^{b_{j}(t)}\left(t-f_{j}(s)\right)^{\beta_{j}} \phi(s) f_{j}^{\prime}(s) d s \\
& \quad=\max _{i=1, \ldots, m}\left\{\int_{b_{i}\left(t_{0}\right)}^{b_{i}(t)}\left(t-f_{i}(s)\right)^{\beta_{i}} \phi(s) f_{i}^{\prime}(s) d s\right\}<\infty .
\end{aligned}
$$

Let

$$
\begin{aligned}
c_{n}(t):= & \left(m \max _{i=1, \ldots, m}\left\{\tilde{h}_{i}(t)\right\}\right)^{n} \frac{\left(\Gamma\left(\beta_{*}+1\right)\right)^{n}}{\Gamma\left(n\left(\beta_{*}+1\right)\right)} \\
& \times\left(t-t_{0}\right)^{(n-1) c\left(\beta_{*}+1\right)} \\
& \times \int_{b_{j}\left(t_{0}\right)}^{b_{j}(t)}\left(t-f_{j}(s)\right)^{\beta_{j}} \phi(s) f_{j}^{\prime}(s) d s .
\end{aligned}
$$

Then $\sum_{n=1}^{\infty} c_{n}(t)$ is convergent on $\left(t_{0}+1, T\right)$ by the ratio test ([25, pages 66-67]) because

$$
\begin{aligned}
\lim _{n \rightarrow+\infty} & \frac{c_{n+1}(t)}{c_{n}(t)} \\
= & \lim _{n \rightarrow+\infty} m \max _{i=1, \ldots, m}\left\{\widetilde{h}_{i}(t)\right\} \\
& \times \frac{\Gamma\left(\beta_{*}+1\right) \Gamma\left(n\left(\beta_{*}+1\right)\right)}{\Gamma\left((n+1)\left(\beta_{*}+1\right)\right)}\left(t-t_{0}\right)^{c\left(\beta_{*}+1\right)} \\
= & \lim _{n \rightarrow+\infty} B\left(\beta_{*}+1, n\left(\beta_{*}+1\right)\right) m \\
& \times \max _{i=1, \ldots, m}\left\{\widetilde{h}_{i}(t)\right\}\left(t-t_{0}\right)^{c\left(\beta_{*}+1\right)} \\
= & \lim _{n \rightarrow+\infty} \frac{\Gamma\left(\beta_{*}+1\right)}{\left(n\left(\beta_{*}+1\right)\right)^{\beta_{*}+1} m} \\
& \times \max _{i=1, \ldots, m}\left\{\widetilde{h}_{i}(t)\right\}\left(t-t_{0}\right)^{c\left(\beta_{*}+1\right)}=0,
\end{aligned}
$$

where we note that $m \max _{i=1, \ldots, m}\left\{\widetilde{h}_{i}(t)\right\}\left(t-t_{0}\right)^{c\left(\beta_{*}+1\right)}$ is a continuous function on $\left[t_{0}, T\right)$ and $\Gamma\left(\beta_{*}+1\right) /\left(n\left(\beta_{*}+1\right)\right)^{\beta_{*}+1}$ is a Stirling's approximation of $B\left(\beta_{*}+1, n\left(\beta_{*}+1\right)\right)$ as $n \rightarrow+\infty$, as known in [26, pages 59]. It implies that $\sum_{n=1}^{\infty} A_{n} \phi(t)$ is convergent on $\left(t_{0}+1, T\right)$. The case of $t \in\left[t_{0}, t_{0}+1\right]$ can be proved similarly. Then, $\sum_{n=1}^{\infty} A_{n} \phi(t)$ is convergent for $\phi \epsilon$ $L_{\text {loc, } w}^{1}\left[t_{0}, T\right)$ and arbitrarily given $t \in\left[t_{0}, T\right)$. Passing to the limit as $n \rightarrow+\infty$ in (34), by (44) we get

$$
\begin{aligned}
& u(t) \leq \sum_{n=0}^{\infty} A_{n} a(t) \\
&=a(t)+\sum_{n=1}^{\infty}\left\{\sum_{1 \leq k_{1}, \ldots, k_{n} \leq m} \frac{\prod_{i=1}^{n} \Gamma\left(\beta_{k_{i}}+1\right) \widetilde{h}_{k_{i}}(t)}{\Gamma\left(\sum_{i=1}^{n} \beta_{k_{i}}+n\right)}\right. \\
& \quad \times \int_{b_{k_{n}}\left(t_{0}\right)}^{b_{k_{n}}(t)}\left(t-f_{k_{n}}(s)\right)^{\sum_{i=1}^{n} \beta_{k_{i}}+n-1}
\end{aligned}
$$

$$
\left.\times f_{k_{n}}^{\prime}(s) a(s) d s\right\} .
$$

Since $k_{i}$ 's are chosen arbitrarily, by interchanging $k_{n}$ with $k_{1}$ in (54), we obtain the estimate (17) and complete the proof of the theorem.

\section{Application to Dependence}

Recently, increasing interest was given to fractional differential equations (see monographs [11, 23]). In this section we consider the Cauchy problem of the general fractional differential equation

$$
\begin{gathered}
D_{t_{0}}^{1} x(t)=\sum_{i=1}^{m} D_{t_{0}}^{\alpha_{i}} f_{i}\left(t, x\left(b_{i}(t)\right)\right), \\
x\left(t_{0}\right)=a,
\end{gathered}
$$


where $0 \leq \alpha_{i}<1, t_{0} \leq t \leq T<+\infty, D_{t_{0}}^{\alpha_{i}}$ is defined as in the Introduction, particularly, $D_{t_{0}}^{1}$ denotes $d / d t$ and $D_{t_{0}}^{0}$ denotes identity map, $b_{i}:\left[t_{0}, T\right] \rightarrow\left[t_{0}, T\right]$ and $f_{i}:\left[t_{0}, T\right] \times \mathbb{R} \rightarrow \mathbb{R}$, $i=1, \ldots, m$. This system includes the system

$$
\begin{gathered}
D_{0}^{1} x(t)+p D_{0}^{\alpha} x(t)+D_{0}^{0} x(t)=q(t), \\
x\left(0^{+}\right)=a,
\end{gathered}
$$

as a special case, which was considered in [27] and corresponds to the Basset problem when $\alpha=1 / 2$, a classical problem in fluid dynamics concerning the unsteady motion of a particle accelerating in a viscous fluid under the action of gravity (see [28]). We will give continuous dependence for solutions of (55) associated with the initial condition (56) on the derivative orders $\alpha_{i}$ 's, the initial data $a$, and the functions $f_{i}$ and $b_{i}$.

Proposition 3. Suppose that each $b_{i} \in C^{1}\left(\left[t_{0}, T\right],\left[t_{0}, T\right]\right)$ is strictly increasing such that $b_{i}(t) \leq t$ and each $f_{i} \in C\left(\left[t_{0}, T\right] \times\right.$ $[a-\rho, a+\rho], \mathbb{R})$ satisfies that $\left|f_{i}\left(t, x_{1}\right)-f_{i}\left(t, x_{2}\right)\right| \leq L_{i}\left|x_{1}-x_{2}\right|$, $i=1, \ldots, m$, where $T>0, \rho>0$, and $L_{i}>0$ are constant. Then, the Cauchy problem (55)-(56) has a unique solution on $\left[t_{0}, t_{0}+h\right]$ for a certain constant $0<h<T$ and the solution depends continuously on $a, \alpha_{i}$ 's, $f_{i}^{\prime}$ 's, and $b_{i}$ 's for all $t \in\left[t_{0}, t_{0}+\right.$ $h$.

Proof. The Cauchy problem (55)-(56) is equivalent to the integral equation

$$
\begin{aligned}
& x(t) \\
& =a+\sum_{i=1}^{m} \int_{t_{0}}^{t} \frac{1}{\Gamma\left(1-\alpha_{i}\right)} d\left(\int_{t_{0}}^{s}(s-\tau)^{-\alpha_{i}} f_{i}\left(\tau, x\left(b_{i}(\tau)\right)\right) d \tau\right) \\
& =a+\sum_{i=1}^{m} \frac{1}{\Gamma\left(1-\alpha_{i}\right)} \int_{t_{0}}^{t}(t-s)^{-\alpha_{i}} f_{i}\left(s, x\left(b_{i}(s)\right)\right) d s .
\end{aligned}
$$

Define a sequence $\left\{\varphi_{n}(t)\right\}$ such that $\varphi_{0}(t) \equiv a$ and

$$
\begin{aligned}
& \varphi_{n}(t)= a+\sum_{i=1}^{m} \frac{1}{\Gamma\left(1-\alpha_{i}\right)} \\
& \times \int_{t_{0}}^{t}(t-s)^{-\alpha_{i}} f_{i}\left(s, \varphi_{n-1}\left(b_{i}(s)\right)\right) d s, \\
& n=1,2, \ldots,
\end{aligned}
$$

where $t \in\left[t_{0}, t_{0}+h\right]$. We first claim that all $\varphi_{n}(t)$ are welldefined and continuous in $\left[t_{0}, t_{0}+h\right]$, such that $\left|\varphi_{n}(t)-a\right| \leq \rho$. In fact, it is true for $n=0$. Suppose that it is true for some $n$. Then, $\varphi_{n+1}$ is also well defined by (59) and continuous in $\left[t_{0}, t_{0}+h\right]$. Moreover,

$$
\begin{aligned}
& \left|\varphi_{n+1}(t)-a\right| \\
& \quad \leq \sum_{i=1}^{m} \frac{1}{\Gamma\left(1-\alpha_{i}\right)} \int_{t_{0}}^{t}(t-s)^{-\alpha_{i}}\left|f_{i}\left(s, \varphi_{n}\left(b_{i}(s)\right)\right)\right| d s \\
& \quad \leq \sum_{i=1}^{m} \frac{M h^{1-\alpha_{i}}}{\Gamma\left(2-\alpha_{i}\right)} \leq \rho,
\end{aligned}
$$

where

$$
\begin{aligned}
& M:=\max _{(t, x) \in\left[\begin{array}{c}
\left.t_{0}, T\right] \times[a-\rho, a+\rho] \\
i=1, \ldots, m
\end{array}\right]}\left|f_{i}(t, x)\right|, \\
& h:=\min \left\{T-t_{0}, \sigma\right\},
\end{aligned}
$$

and $\sigma$ is a positive constant such that

$$
\sum_{i=1}^{m} \frac{M \sigma^{1-\alpha_{i}}}{\Gamma\left(2-\alpha_{i}\right)}=\rho
$$

The existence of $\sigma$ is guaranteed by the intermediate theorem for continuous functions. Thus, the claim is proved by induction for all $n$.

The convergence of the sequence $\left\{\varphi_{n}(t)\right\}$ is equivalent to the convergence of the series $\varphi_{0}(t)+\sum_{l=1}^{\infty}\left(\varphi_{l}(t)-\varphi_{l-1}(t)\right)$. We claim that

$$
\begin{aligned}
& \left|\varphi_{l}(t)-\varphi_{l-1}(t)\right| \\
& \quad \leq \sum_{1 \leq k_{1}, \ldots, k_{l} \leq m} \frac{M \prod_{i=2}^{l} L_{k_{i}}}{\Gamma\left(1+\sum_{i=1}^{l}\left(1-\alpha_{k_{i}}\right)\right)}\left(t-t_{0}\right)^{\sum_{i=1}^{l}\left(1-\alpha_{k_{i}}\right)},
\end{aligned}
$$

for all $t \in\left[t_{0}, t_{0}+h\right]$ and all integers $l$. It can be checked easily for $l=1$. Suppose that it is true for some $l$. By changing $t$ into $b_{k_{l+1}}(s)$ in (63), we get from $b_{k_{l+1}}(s) \leq s$ that

$$
\begin{aligned}
& \left|\varphi_{l}\left(b_{k_{l+1}}(s)\right)-\varphi_{l-1}\left(b_{k_{l+1}}(s)\right)\right| \\
& \leq \sum_{1 \leq k_{1}, \ldots, k_{l} \leq m} \frac{M \prod_{i=2}^{l} L_{k_{i}}}{\Gamma\left(1+\sum_{i=1}^{l}\left(1-\alpha_{k_{i}}\right)\right)} \\
& \quad \times\left(b_{k_{l+1}}(s)-t_{0}\right)^{\sum_{i=1}^{l}\left(1-\alpha_{k_{i}}\right)} \\
& \leq \sum_{1 \leq k_{1}, \ldots, k_{l} \leq m} \frac{M \prod_{i=2}^{l} L_{k_{i}}}{\Gamma\left(1+\sum_{i=1}^{l}\left(1-\alpha_{k_{i}}\right)\right)} \\
& \quad \times\left(s-t_{0}\right)^{\sum_{i=1}^{l}\left(1-\alpha_{k_{i}}\right) .}
\end{aligned}
$$

Combining with (59), we have

$$
\begin{aligned}
& \left|\varphi_{l+1}(t)-\varphi_{l}(t)\right| \\
& \leq \sum_{k_{l+1}=1}^{m} \frac{1}{\Gamma\left(1-\alpha_{k_{l+1}}\right)} \\
& \quad \times \int_{t_{0}}^{t}(t-s)^{-\alpha_{k_{l+1}}} \\
& \quad \times \mid f_{k_{l+1}}\left(s, \varphi_{l}\left(b_{k_{l+1}}(s)\right)\right) \\
& \quad-f_{k_{l+1}}\left(s, \varphi_{l-1}\left(b_{k_{l+1}}(s)\right)\right) \mid d s
\end{aligned}
$$




$$
\begin{aligned}
& \leq \sum_{k_{l+1}=1}^{m} \frac{L_{k_{l+1}}}{\Gamma\left(1-\alpha_{k_{l+1}}\right)} \\
& \times \int_{t_{0}}^{t}(t-s)^{-\alpha_{k_{l+1}}}\left|\varphi_{l}\left(b_{k_{l+1}}(s)\right)-\varphi_{l-1}\left(b_{k_{l+1}}(s)\right)\right| d s \\
& \leq \sum_{1 \leq k_{1}, \ldots, k_{l+1} \leq m} \frac{M \prod_{i=2}^{l+1} L_{k_{i}}}{\Gamma\left(1+\sum_{i=1}^{l}\left(1-\alpha_{k_{i}}\right)\right) \Gamma\left(1-\alpha_{k_{l+1}}\right)} \\
& \times \int_{t_{0}}^{t}(t-s)^{-\alpha_{k_{l+1}}}\left(s-t_{0}\right)^{\sum_{i=1}^{l}\left(1-\alpha_{k_{i}}\right)} d s \\
& =\sum_{1 \leq k_{1}, \ldots, k_{l+1} \leq m}\left(M\left(\prod_{i=2}^{l+1} L_{k_{i}}\right)\right. \\
& \times B\left(1+\sum_{i=1}^{l}\left(1-\alpha_{k_{i}}\right), 1-\alpha_{k_{l+1}}\right) \\
& \times\left(\Gamma\left(1+\sum_{i=1}^{l}\left(1-\alpha_{k_{i}}\right)\right)\right. \\
& \left.\left.\times \Gamma\left(1-\alpha_{k_{l+1}}\right)\right)^{-1}\right) \\
& \times\left(t-t_{0}\right)^{\sum_{i=1}^{l+1}\left(1-\alpha_{k_{i}}\right)} \\
& =\sum_{1 \leq k_{1}, \ldots, k_{l+1} \leq m} \frac{M \prod_{i=2}^{l+1} L_{k_{i}}}{\Gamma\left(1+\sum_{i=1}^{l+1}\left(1-\alpha_{k_{i}}\right)\right)}\left(t-t_{0}\right)^{\sum_{i=1}^{l+1}\left(1-\alpha_{k_{i}}\right)} .
\end{aligned}
$$

Thus, (63) holds for $l+1$ and the claim is proved for all $l$ by induction. From (63), for $t \leq t_{0}+h$ we have

$$
\begin{aligned}
\left|\varphi_{l}(t)-\varphi_{l-1}(t)\right| & \leq \sum_{1 \leq k_{1}, \ldots, k_{l} \leq m} \frac{M h^{\sum_{i=1}^{l}\left(1-\alpha_{k_{i}}\right)} \prod_{i=2}^{l} L_{k_{i}}}{\Gamma\left(1+\sum_{i=1}^{l}\left(1-\alpha_{k_{i}}\right)\right)} \\
& \leq \frac{m^{l} M h^{l\left(1-\alpha_{*}\right)} L^{l-1}}{\Gamma\left(1+l\left(1-\alpha^{*}\right)\right)},
\end{aligned}
$$

where $L:=\max _{1 \leq i \leq m} L_{i}, \alpha^{*}:=\max _{1 \leq i \leq m} \alpha_{i}$, and $\alpha_{*}:=$ $\min _{1 \leq i \leq m} \alpha_{i}$. By the ratio test ([25, pp. 66-67]), we get that $\sum_{l=1}^{\infty} m^{l} M h^{l\left(1-\alpha_{*}\right)} L^{l-1} / \Gamma\left(1+l\left(1-\alpha^{*}\right)\right)$ is convergent, implying that the series $\varphi_{0}(t)+\sum_{l=1}^{\infty}\left(\varphi_{l}(t)-\varphi_{l-1}(t)\right)$ and therefore the sequence $\left\{\varphi_{n}(t)\right\}$ are uniformly convergent in $\left[t_{0}, t_{0}+h\right]$. Let $\varphi(t):=\lim _{n \rightarrow \infty} \varphi_{n}(t)$, which is well-defined and continuous in $\left[t_{0}, t_{0}+h\right]$ such that $|\varphi(t)-a| \leq \rho$. One can check that $\varphi$ is a continuous solution of the Cauchy problem (55)-(56) in $\left[t_{0}, t_{0}+h\right]$.
Next, we prove the continuous dependence of the solution. Consider the Cauchy problem

$$
\begin{gathered}
D_{t_{0}}^{1} x(t)=\sum_{i=1}^{m} D_{t_{0}}^{\widetilde{\alpha}_{i}} \widetilde{f}_{i}\left(t, x\left(\widetilde{b}_{i}(t)\right)\right), \\
x\left(t_{0}\right)=\tilde{a},
\end{gathered}
$$

where $0 \leq \widetilde{\alpha}_{i}<1, t_{0} \leq t \leq T<+\infty, \widetilde{b}_{i} \in C^{1}\left(\left[t_{0}, T\right],\left[t_{0}, T\right]\right)$ is strictly increasing such that $b_{i}(t) \leq t$, and $\tilde{f}_{i} \in C\left(\left[t_{0}, T\right] \times\right.$ $[\tilde{a}-\rho, \tilde{a}+\rho], \mathbb{R})$ is Lipschitzian in the second variable with the Lipschitz constant $\widetilde{L}_{i}, i=1, \ldots, m$. As above, we similarly obtain a solution $\psi$ of the Cauchy problem (67)-(68). Similar to (58), we have

$$
\begin{aligned}
& \varphi(t)=a+\sum_{i=1}^{m} \frac{1}{\Gamma\left(1-\alpha_{i}\right)} \int_{t_{0}}^{t}(t-s)^{-\alpha_{i}} f_{i}\left(s, \varphi\left(b_{i}(s)\right)\right) d s, \\
& \psi(t)=\widetilde{a}+\sum_{i=1}^{m} \frac{1}{\Gamma\left(1-\widetilde{\alpha}_{i}\right)} \int_{t_{0}}^{t}(t-s)^{-\widetilde{\alpha}_{i}} \widetilde{f}_{i}\left(s, \psi\left(\widetilde{b}_{i}(s)\right)\right) d s .
\end{aligned}
$$

It follows that

$$
\begin{aligned}
& |\varphi(t)-\psi(t)| \\
& \leq|a-\widetilde{a}|+\sum_{i=1}^{m} \mid \frac{1}{\Gamma\left(1-\alpha_{i}\right)} \\
& \times \int_{t_{0}}^{t}(t-s)^{-\alpha_{i}} f_{i}\left(s, \varphi\left(b_{i}(s)\right)\right) d s \\
& -\frac{1}{\Gamma\left(1-\tilde{\alpha}_{i}\right)} \\
& \times \int_{t_{0}}^{t}(t-s)^{-\widetilde{\alpha}_{i}} \widetilde{f}_{i}\left(s, \psi\left(\widetilde{b}_{i}(s)\right)\right) d s \mid \\
& \leq \Xi(t)+\sum_{i=1}^{m}\left\{\mid \frac{1}{\Gamma\left(1-\alpha_{i}\right)}\right. \\
& \times \int_{t_{0}}^{t}(t-s)^{-\alpha_{i}} f_{i}\left(s, \varphi\left(b_{i}(s)\right)\right) d s \\
& -\frac{1}{\Gamma\left(1-\alpha_{i}\right)} \\
& \left.\times \int_{t_{0}}^{t}(t-s)^{-\alpha_{i}} f_{i}\left(s, \psi\left(b_{i}(s)\right)\right) d s \mid\right\} \\
& \leq \Xi(t)+\sum_{i=1}^{m} \frac{L_{i}}{\Gamma\left(1-\alpha_{i}\right)} \\
& \times \int_{b_{i}\left(t_{0}\right)}^{b_{i}(t)}\left(t-b_{i}^{-1}(s)\right)^{-\alpha_{i}}|\varphi(s)-\psi(s)| d\left(b_{i}^{-1}(s)\right),
\end{aligned}
$$


where

$\Xi(t)$

$$
\begin{aligned}
& :=|a-\tilde{a}| \\
& +\sum_{i=1}^{m}\left\{\mid \frac{1}{\Gamma\left(1-\alpha_{i}\right)}\right. \\
& \times \int_{t_{0}}^{t}(t-s)^{-\alpha_{i}} f_{i}\left(s, \psi\left(b_{i}(s)\right)\right) d s \\
& -\frac{1}{\Gamma\left(1-\tilde{\alpha}_{i}\right)} \\
& \times \int_{t_{0}}^{t}(t-s)^{-\widetilde{\alpha}_{i}} f_{i}\left(s, \psi\left(b_{i}(s)\right)\right) d s \\
& +\mid \frac{1}{\Gamma\left(1-\tilde{\alpha}_{i}\right)} \int_{t_{0}}^{t}(t-s)^{-\widetilde{\alpha}_{i}} f_{i}\left(s, \psi\left(b_{i}(s)\right)\right) d s \\
& -\frac{1}{\Gamma\left(1-\tilde{\alpha}_{i}\right)} \int_{t_{0}}^{t}(t-s)^{-\widetilde{\alpha}_{i}} \widetilde{f}_{i}\left(s, \psi\left(b_{i}(s)\right)\right) d s \mid \\
& +\mid \frac{1}{\Gamma\left(1-\tilde{\alpha}_{i}\right)} \int_{t_{0}}^{t}(t-s)^{-\widetilde{\alpha}_{i}} \widetilde{f}_{i}\left(s, \psi\left(b_{i}(s)\right)\right) d s \\
& \left.-\frac{1}{\Gamma\left(1-\widetilde{\alpha}_{i}\right)} \int_{t_{0}}^{t}(t-s)^{-\widetilde{\alpha}_{i}} \widetilde{f}_{i}\left(s, \psi\left(\widetilde{b}_{i}(s)\right)\right) d s \mid\right\}
\end{aligned}
$$$$
\leq|a-\widetilde{a}|
$$$$
\begin{gathered}
+\sum_{i=1}^{m}\left\{\left|\frac{\left(t-t_{0}\right)^{1-\alpha_{i}}}{\Gamma\left(2-\alpha_{i}\right)}-\frac{\left(t-t_{0}\right)^{1-\widetilde{\alpha}_{i}}}{\Gamma\left(2-\widetilde{\alpha}_{i}\right)}\right|\left\|f_{i}\right\|\right. \\
+\frac{\left(t-t_{0}\right)^{1-\widetilde{\alpha}_{i}}}{\Gamma\left(2-\widetilde{\alpha}_{i}\right)}\left\|f_{i}-\widetilde{f}_{i}\right\| \\
+\frac{\widetilde{L}_{i}}{\Gamma\left(1-\widetilde{\alpha}_{i}\right)} \int_{t_{0}}^{t}(t-s)^{-\widetilde{\alpha}_{i}}
\end{gathered}
$$$$
\left.\times\left|\psi\left(b_{i}(s)\right)-\psi\left(\widetilde{b}_{i}(s)\right)\right| d s\right\}
$$

$\left\|f_{i}\right\|:=\max _{(t, x) \in\left[t_{0}, T\right] \times[a-\rho, a+\rho]}\left|f_{i}(t, x)\right|$, and $\left\|f_{i}-\widetilde{f}_{i}\right\|:=$ $\max _{(t, x) \in\left[t_{0}, T\right] \times[a-\rho, a+\rho]}\left|f_{i}(t, x)-\widetilde{f}_{i}(t, x)\right|$. Thus, applying the inequality given in Corollary 2 to $(70)$, we obtain that

$$
\begin{array}{r}
|\varphi(t)-\psi(t)| \leq \widetilde{\Xi}(t) \prod_{i=1}^{m} E_{1-\alpha_{i}}\left(L_{i}\left(t-t_{0}\right)^{1-\alpha_{i}}\right), \\
\forall t \in\left[t_{0}, t_{0}+h\right],
\end{array}
$$

where $\widetilde{\Xi}(t):=\max _{t_{0} \leq \tau \leq t} \Xi(\tau)$.
Note from (71) that $\widetilde{\Xi}(t)$ contains $\mid\left(t-t_{0}\right)^{1-\alpha_{i}} / \Gamma\left(2-\alpha_{i}\right)-$ $\left(t-t_{0}\right)^{1-\widetilde{\alpha}_{i}} / \Gamma\left(2-\widetilde{\alpha}_{i}\right) \mid$ and $\left|\psi\left(b_{i}(s)\right)-\psi\left(\widetilde{b}_{i}(s)\right)\right|$, which will be estimated with $\left\|b_{i}-\widetilde{b}_{i}\right\|:=\max _{t_{0} \leq \tau \leq t_{0}+h}\left|b_{i}(\tau)-\widetilde{b}_{i}(\tau)\right|$ and $\left|\alpha_{i}-\widetilde{\alpha}_{i}\right|$, respectively. In (71) we have $\left\|f_{i}\right\| \leq M$ in (61). Since $\left(t-t_{0}\right)^{1-\alpha_{i}}$ and $\Gamma\left(2-\alpha_{i}\right)$ are both $C^{1}$ functions in $\alpha_{i} \in[0,1]$, and $\Gamma\left(2-\alpha_{i}\right)>0$ for all $\alpha_{i} \in[0,1]$, we see that $\left(t-t_{0}\right)^{1-\alpha_{i}} / \Gamma(2-$ $\left.\alpha_{i}\right)$ is $C^{1}$ in $\alpha_{i} \in[0,1]$. By the Lagrange mean value theorem (see [25, page 108]), function $\left(t-t_{0}\right)^{1-\alpha_{i}} / \Gamma\left(2-\alpha_{i}\right)$ satisfies the Lipschitz condition uniformly with respect to $t \in\left[t_{0}, t_{0}+h\right]$; that is, there exists a constant $N>0$ such that

$$
\left|\frac{\left(t-t_{0}\right)^{1-\alpha_{i}}}{\Gamma\left(2-\alpha_{i}\right)}-\frac{\left(t-t_{0}\right)^{1-\widetilde{\alpha}_{i}}}{\Gamma\left(2-\widetilde{\alpha}_{i}\right)}\right| \leq N\left|\alpha_{i}-\widetilde{\alpha}_{i}\right|, \quad \forall \alpha_{i}, \widetilde{\alpha}_{i} \in[0,1]
$$

On the other hand, from the equivalent integral equation of (67)

$$
\begin{aligned}
\psi(t)= & \psi\left(t_{0}\right) \\
& +\sum_{j=1}^{m} \frac{1}{\Gamma\left(1-\tilde{\alpha}_{j}\right)} \int_{t_{0}}^{t}(t-s)^{-\widetilde{\alpha}_{j}} \widetilde{f}_{j}\left(s, \psi\left(\widetilde{b}_{j}(s)\right)\right) d s,
\end{aligned}
$$

we get

$$
\left|\psi(t)-\psi\left(t_{0}\right)\right| \leq \sum_{j=1}^{m} \frac{\widetilde{M}}{\Gamma\left(2-\widetilde{\alpha}_{j}\right)}\left|t-t_{0}\right|^{1-\widetilde{\alpha}_{j}},
$$

where

$$
\widetilde{M}:=\max _{\substack{(t, x) \in\left[t_{0}, T\right] \times[a-\rho, a+\rho] \\ j=1, \ldots, m}}\left|\widetilde{f}_{j}(t, x)\right| .
$$

Choosing $t=b_{i}(s)$ and $t_{0}=\widetilde{b}_{i}(s)$ in (74), we obtain

$$
\begin{aligned}
\left|\psi\left(b_{i}(s)\right)-\psi\left(\widetilde{b}_{i}(s)\right)\right| & \leq \sum_{j=1}^{m} \frac{\widetilde{M}}{\Gamma\left(2-\widetilde{\alpha}_{j}\right)}\left|b_{i}(s)-\widetilde{b}_{i}(s)\right|^{1-\widetilde{\alpha}_{j}} \\
& \leq \sum_{j=1}^{m} \frac{\widetilde{M}}{\Gamma\left(2-\widetilde{\alpha}_{j}\right)}\left\|b_{i}-\widetilde{b}_{i}\right\|^{1-\widetilde{\alpha}_{j}} .
\end{aligned}
$$

Summarily, from (71), (73) and (75) we get

$$
\begin{aligned}
& \Xi(t) \leq|a-\tilde{a}| \\
& +\sum_{i=1}^{m}\left\{M N\left|\alpha_{i}-\widetilde{\alpha}_{i}\right|+\frac{\left(t-t_{0}\right)^{1-\widetilde{\alpha}_{i}}}{\Gamma\left(2-\widetilde{\alpha}_{i}\right)}\left\|f_{i}-\widetilde{f}_{i}\right\|\right. \\
& +\frac{\widetilde{L}_{i}}{\Gamma\left(1-\widetilde{\alpha}_{i}\right)} \int_{t_{0}}^{t}(t-s)^{-\widetilde{\alpha}_{i}} \sum_{j=1}^{m} \frac{\widetilde{M}}{\Gamma\left(2-\widetilde{\alpha}_{j}\right)} \\
& \left.\times\left\|b_{i}-\widetilde{b}_{i}\right\|^{1-\widetilde{\alpha}_{j}} d s\right\}
\end{aligned}
$$




$$
\begin{array}{r}
\leq|a-\widetilde{a}|+\sum_{i=1}^{m}\left\{M N\left|\alpha_{i}-\widetilde{\alpha}_{i}\right|+\frac{h^{1-\widetilde{\alpha}_{i}}}{\Gamma\left(2-\widetilde{\alpha}_{i}\right)}\left\|f_{i}-\widetilde{f}_{i}\right\|\right. \\
\left.+\frac{\widetilde{M} \widetilde{L}_{i} h^{1-\widetilde{\alpha}_{i}}}{\Gamma\left(2-\widetilde{\alpha}_{i}\right)} \sum_{j=1}^{m} \frac{\left\|b_{i}-\widetilde{b}_{i}\right\|^{1-\widetilde{\alpha}_{j}}}{\Gamma\left(2-\widetilde{\alpha}_{j}\right)}\right\} .
\end{array}
$$

It follows from (72) that

$$
\begin{aligned}
\|\varphi-\psi\| \leq & \prod_{i=1}^{m} E_{1-\alpha_{i}}\left(L_{i} h^{1-\alpha_{i}}\right)|a-\widetilde{a}| \\
& +M N \prod_{i=1}^{m} E_{1-\alpha_{i}}\left(L_{i} h^{1-\alpha_{i}}\right) \sum_{i=1}^{m}\left|\alpha_{i}-\widetilde{\alpha}_{i}\right| \\
& +\prod_{i=1}^{m} E_{1-\alpha_{i}}\left(L_{i} h^{1-\alpha_{i}}\right) \sum_{i=1}^{m} \frac{h^{1-\widetilde{\alpha}_{i}}}{\Gamma\left(2-\widetilde{\alpha}_{i}\right)}\left\|f_{i}-\widetilde{f}_{i}\right\| \\
& +\widetilde{M} \prod_{i=1}^{m} E_{1-\alpha_{i}}\left(L_{i} h^{1-\alpha_{i}}\right) \\
& \times \sum_{1 \leq i, j \leq m} \frac{\widetilde{L}_{i} h^{1-\widetilde{\alpha}_{i}}}{\Gamma\left(2-\widetilde{\alpha}_{i}\right) \Gamma\left(2-\widetilde{\alpha}_{j}\right)}\left\|b_{i}-\widetilde{b}_{i}\right\|^{1-\widetilde{\alpha}_{j}} ;
\end{aligned}
$$

that is, the solution $\varphi$ of the Cauchy problem (55)-(56) depends continuously on $a, \alpha_{i}, f_{i}$ and $b_{i}$.

As a complement, we note from (79) that $\varphi \equiv \psi$ in $\left[t_{0}, t_{0}+\right.$ $h]$ if $a=\widetilde{a}, \alpha_{i}=\widetilde{\alpha}_{i}, f_{i} \equiv \widetilde{f}_{i}$, and $b_{i} \equiv \widetilde{b}_{i}$. This implies the uniqueness of the solution.

\section{Conflict of Interests}

The author declares that there is no conflict of interests regarding the publication of this paper.

\section{Acknowledgment}

The author is very grateful to Professor Weinian Zhang for his valuable suggestions.

\section{References}

[1] T. H. Gronwall, "Note on the derivatives with respect to a parameter of the solutions of a system of differential equations," Annals of Mathematics, vol. 20, no. 4, pp. 292-296, 1919.

[2] R. Bellman, "The stability of solutions of linear differential equations," Duke Mathematical Journal, vol. 10, pp. 643-647, 1943.

[3] I. A. Bihari, "A generalization of a lemma of Bellman and its application to uniqueness problems of differential equations," Acta Mathematica Academiae Scientiarum Hungaricae, vol. 7, pp. 81-94, 1956.

[4] O. Lipovan, "A retarded Gronwall-like inequality and its applications," Journal of Mathematical Analysis and Applications, vol. 252, no. 1, pp. 389-401, 2000.
[5] R. P. Agarwal, S. Deng, and W. Zhang, "Generalization of a retarded Gronwall-like inequality and its applications," Applied Mathematics and Computation, vol. 165, no. 3, pp. 599-612, 2005.

[6] S. D. Borysenko, M. Ciarletta, and G. Iovane, "Integro-sum inequalities and motion stability of systems with impulse perturbations," Nonlinear Analysis: Theory, Methods \& Applications, vol. 62, no. 3, pp. 417-428, 2005.

[7] W.-S. Cheung, "Some new nonlinear inequalities and applications to boundary value problems," Nonlinear Analysis: Theory, Methods \& Applications, vol. 64, no. 9, pp. 2112-2128, 2006.

[8] W.-S. Wang and X. L. Zhou, "A generalized Gronwall-BellmanOu-Iang type inequality for discontinuous functions and applications to BVP," Applied Mathematics and Computation, vol. 216, no. 11, pp. 3335-3342, 2010.

[9] B. G. Pachpatte, Inequalities for Differential and Integral Equations, vol. 197 of Mathematics in Science and Engineering, Academic Press, New York, NY, USA, 1998.

[10] E. M. Stein, Harmonic Analysis, vol. 43 of Princeton Mathematical Series, Princeton University Press, Princeton, NJ, USA, 1993.

[11] I. Podlubny, Fractional Differential Equations, vol. 198 of Mathematics in Science and Engineering, Academic Press, New York, NY, USA, 1999.

[12] D. Henry, Geometric Theory of Semilinear Parabolic Equations, vol. 840 of Lecture Notes in Mathematics, Springer, Berlin, Germany, 1981.

[13] H. Sano and N. Kunimatsu, "Modified Gronwall's inequality and its application to stabilization problem for semilinear parabolic systems," Systems \& Control Letters, vol. 22, no. 2, pp. 145-156, 1994.

[14] H. P. Ye, J. M. Gao, and Y. S. Ding, "A generalized Gronwall inequality and its application to a fractional differential equation," Journal of Mathematical Analysis and Applications, vol. 328, no. 2, pp. 1075-1081, 2007.

[15] M. Medved', "A new approach to an analysis of Henry type integral inequalities and their Bihari type versions," Journal of Mathematical Analysis and Applications, vol. 214, no. 2, pp. 349366, 1997.

[16] Q. H. Ma and E. H. Yang, "Estimates on solutions of some weakly singular Volterra integral inequalities," Acta Mathematicae Applicatae Sinica, vol. 25, no. 3, pp. 505-515, 2002.

[17] Q. H. Ma and J. Pečarić, "Some new explicit bounds for weakly singular integral inequalities with applications to fractional differential and integral equations," Journal of Mathematical Analysis and Applications, vol. 341, no. 2, pp. 894-905, 2008.

[18] S. B. Hadid and Y. F. Luchko, "An operational method for solving fractional differential equations of an arbitrary real order," Panamerican Mathematical Journal, vol. 6, no. 1, pp. 5773, 1996.

[19] G. M. Mittag-Leffler, "Sur la nouvelle fonction $E_{\alpha}(x)$," Comptes Rendus de l’Académie des Sciences, vol. 137, pp. 554-558, 1903.

[20] A. Wiman, "Über den Fundamentalsatz in der Teorie der Funktionen $E_{\alpha}(x)$," Acta Mathematica, vol. 29, no. 1, pp. 191201, 1905.

[21] T. R. Prabhakar, "A singular integral equation with a generalized Mittag Leffler function in the kernel," Yokohama Mathematical Journal, vol. 19, pp. 7-15, 1971.

[22] A. A. Kilbas and M. Saigo, "On solution of integral equation of Abel-Volterra type," Differential and Integral Equations, vol. 8, no. 5, pp. 993-1011, 1995. 
[23] A. A. Kilbas, H. M. Srivastava, and J. J. Trujillo, Theory and Applications of Fractional Differential Equations, North-Holland Mathematics Studies 204, North-Holland, Amsterdam, The Netherlands, 1981.

[24] E. D. Rainville, Special Functions, The Macmillan Company, New York, NY, USA, 1960.

[25] W. Rudin, Principles of Mathematical Analysis, McGraw-Hill, New York, NY, USA, 3rd edition, 1976.

[26] R. Remmert, Classical Topics in Complex Function Theory, Springer, New York, NY, USA, 1997.

[27] R. Gorenflo and F. Mainardi, "Fractional calculus: integral and differential equations of fractional order," in Fractals and Fractional Calculus in Continuum Mechanics, A. Carpinteri and F. Mainardi, Eds., vol. 378, pp. 223-276, Springer, New York, NY, USA, 1997.

[28] F. Mainardi, "Fractional calculus: some basic problems in continuum and statistical mechanics," in Fractals and Fractional Calculus in Continuum Mechanics, A. Carpinteri and F. Mainardi, Eds., vol. 378, pp. 291-348, Springer, New York, NY, USA, 1997. 


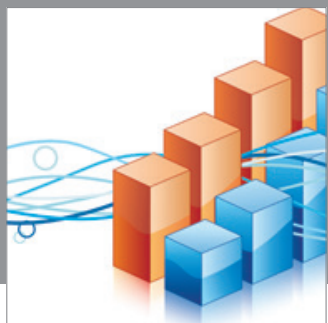

Advances in

Operations Research

mansans

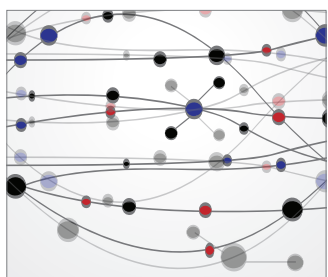

The Scientific World Journal
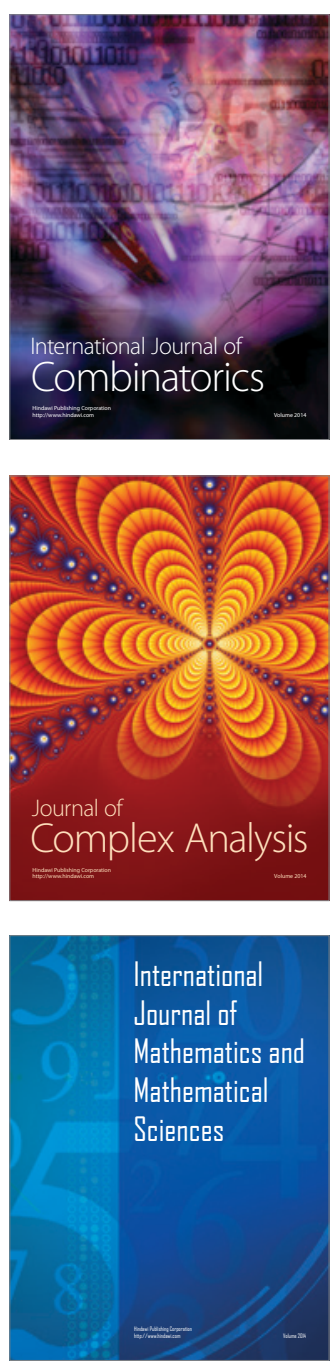
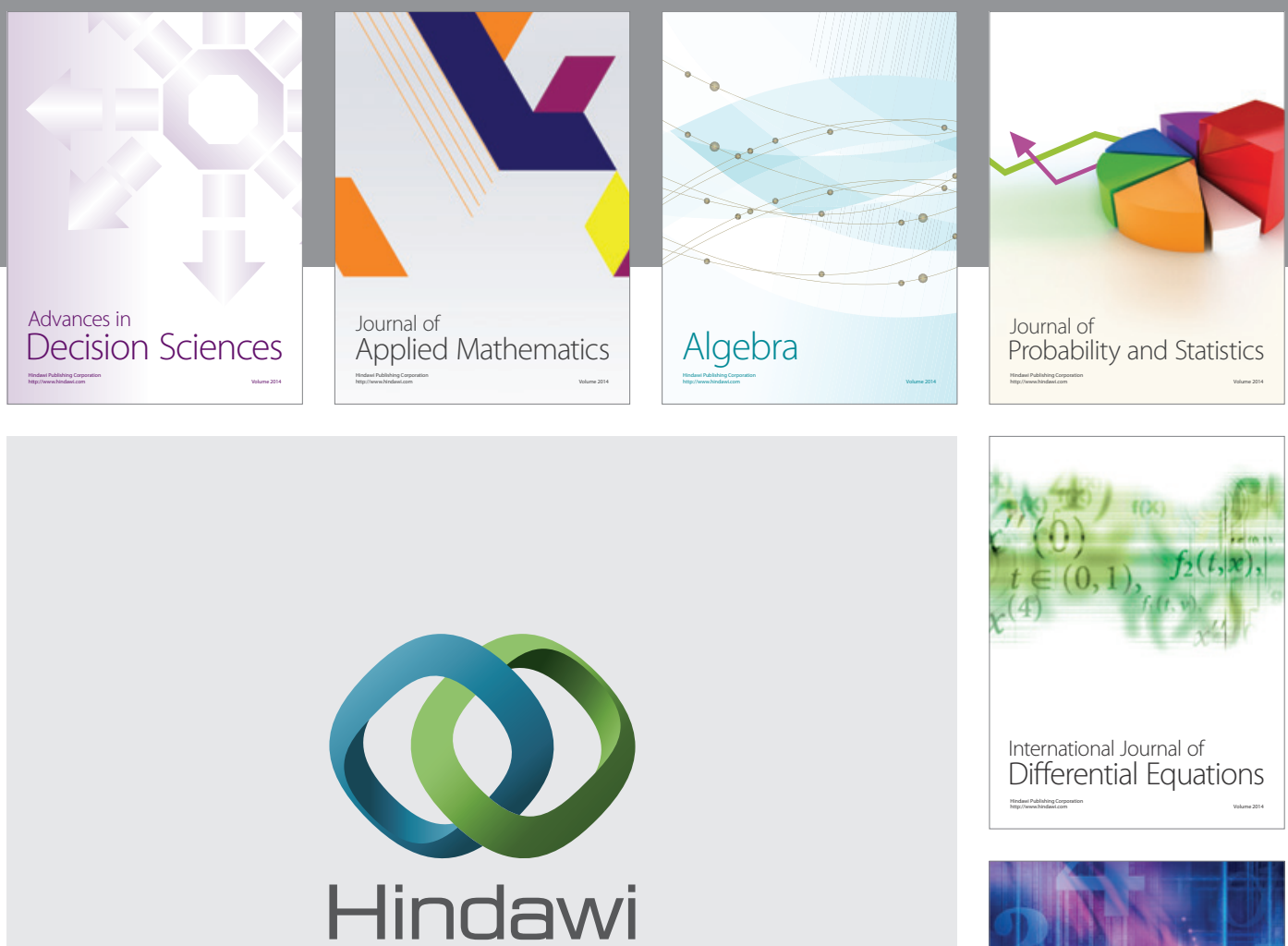

Submit your manuscripts at http://www.hindawi.com
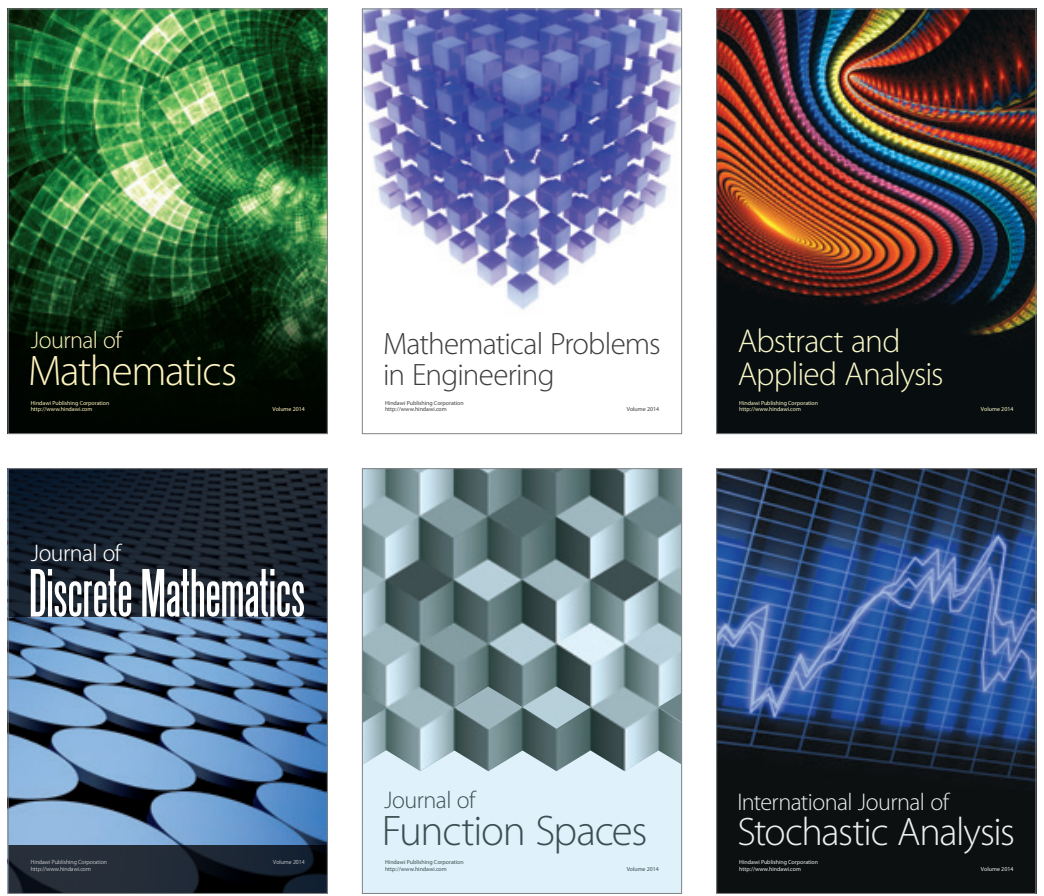

Journal of

Function Spaces

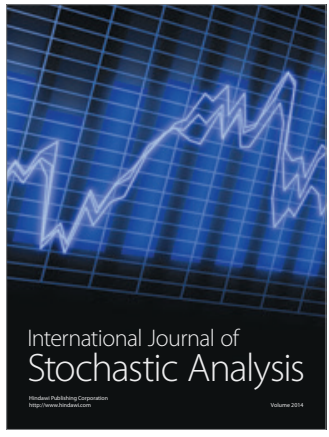

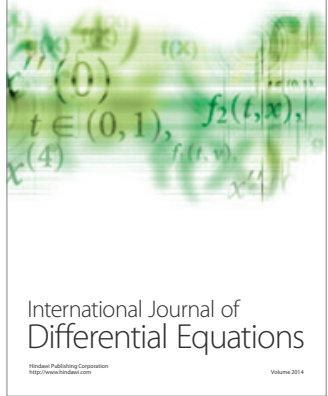
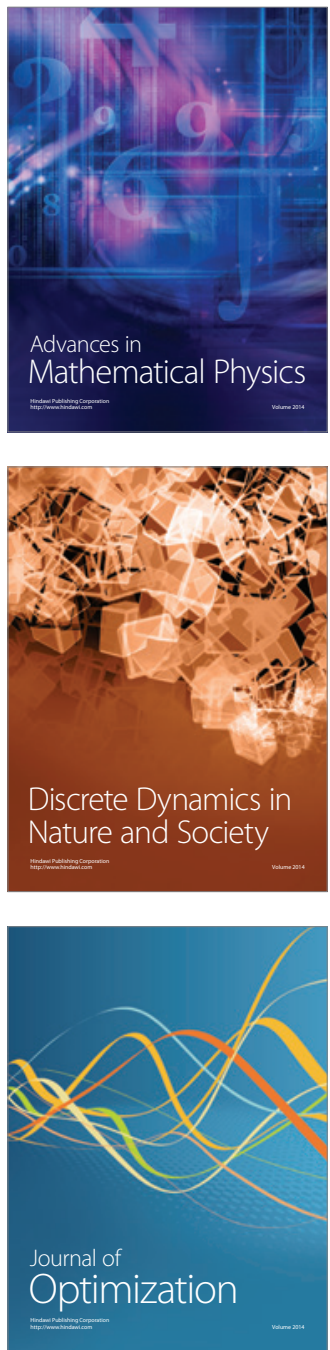